\title{
Photoredox Generation of Sulfonyl Radicals and Coupling with Electron Deficient Olefins
}

\author{
Yiding Chen, Nicole McNamara, Oliver May, Thanigaimalai Pillaiyar, David C. Blakemore, \\ and Steven V. Ley*
}

Cite This: Org. Lett. 2020, 22, 5746-5748

Read Online

\section{ACCESS \\ 山ll Metrics \& More \\ Article Recommendations \\ Supporting Information}

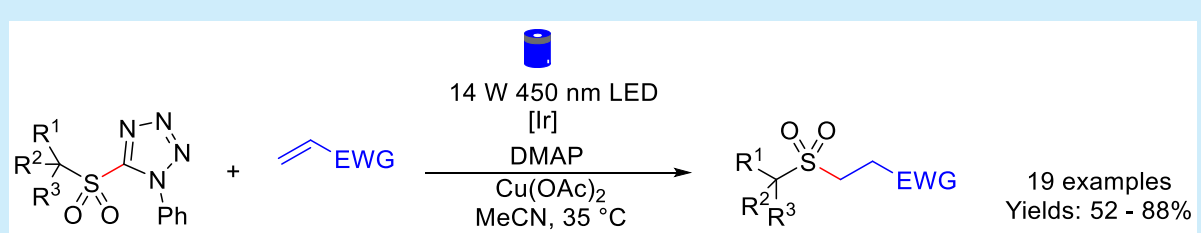

ABSTRACT: Various sulfone tetrazoles were activated via iridium photoredox catalysis in the presence of DMAP to give dialkyl sulfones. The presumed sulfone radical intermediates were trapped by a range of electron-deficient olefins in generally good to excellent yields.

$\mathrm{C}$ ompared to carbon-carbon bond formation using photocatalytic methods, ${ }^{1}$ carbon-sulfur coupling processes are less well-known ${ }^{2}$ but are growing in popularity owing to their presence in pharmaceutical and agrochemical agents. ${ }^{3}$ Notable examples include the photoredox activation of sulfonyl chlorides ${ }^{4}$ and derivatives, ${ }^{5}$ aryl sulfinates, ${ }^{6}$ or sulfinic acids. Various other sulfinates have also been used as radical precursors driving photoredox catalytic events. Recently, Gouverneur et al. developed a silyl-mediated sulfonamide and sulfone preparation from sulfonyl chloride precursors using photoredox methods. ${ }^{8}$

Despite these studies, access to and applications of sulfonyl radicals in cross-coupling reactions are rare. ${ }^{9}$ Here, we report an approach that utilizes photoredox activation of sulfonesubstituted tetrazoles for the generation of sulfonyl radicals that are subsequently intercepted and trapped by electrondeficient olefins. Previously, sulfone-substituted tetrazoles have been featured most notably as partners in the Julia/Kocienski olefination process, ${ }^{10}$ while recently they were used in nickelcatalyzed cross-coupling with organozinc reagents via reductive desulfonylation through a single-electron transfer mechanism. ${ }^{11}$ Interestingly in a preliminary experiment we found the sulfone moiety is retained in the product where sulfone-substituted $\mathrm{N}$ phenyl tetrazole (1a) was subjected to an iridium catalyst system with blue LED irradiation using ethyl acrylate $(\mathbf{2 a})$ as a radical trap to give compound $3 \mathbf{a}$ in modest yield (Scheme 1$)^{12}$

This reaction was quickly optimized which established $\left(\operatorname{Ir}\left[\mathrm{dF}\left(\mathrm{CF}_{3}\right) \mathrm{ppy}\right]_{2}(\mathrm{dtbpy})\right) \mathrm{PF}_{6}$ as the best photocatalyst, 4(dimethylamino)pyridine (DMAP) as an essential component and $\mathrm{Cu}(\mathrm{OAc})_{2}$ was added as an oxidant, with acetonitrile solvent and $14 \mathrm{~W} 420 \mathrm{~nm}$ blue LED light source (Table 1, entry 1). Other photocatalysts such as $\mathrm{Ru}(\mathrm{bpy})_{3} \mathrm{Cl}_{2}$ were ineffective (entry 2). Lewis acid catalyst $\mathrm{Sc}(\mathrm{OTf})_{3}$ did not offer any useful
Scheme 1

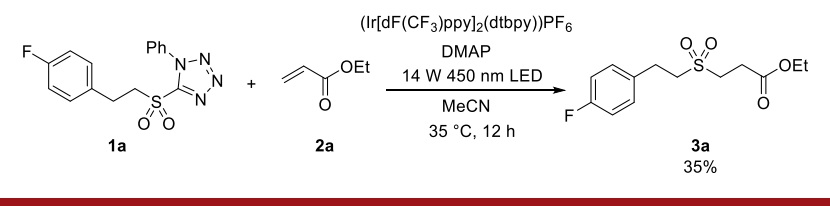

Table 1. Reaction Optimization ${ }^{a}$

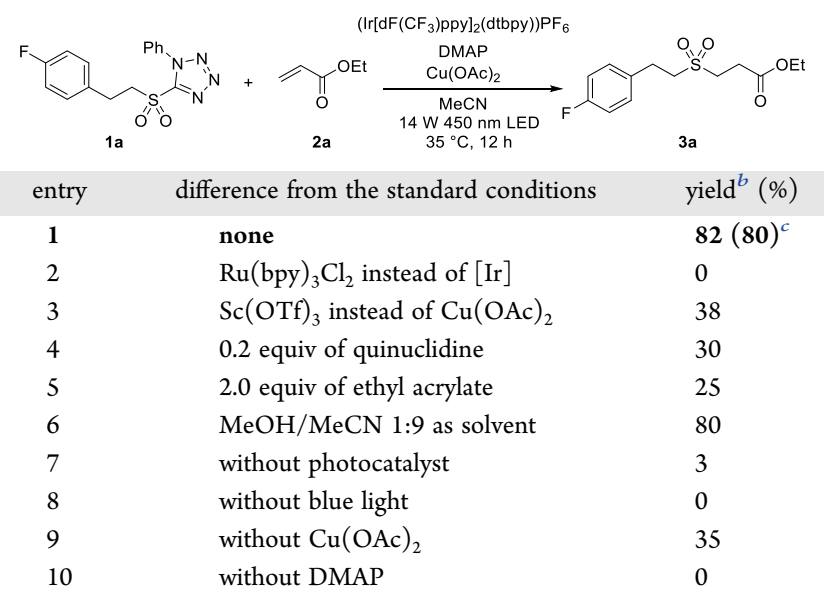

${ }^{a}$ Reaction conditions: $0.1 \mathrm{mmol}, 1.0$ equiv of sulfonyl tetrazole, 5.0 equiv of ethyl acrylate, $2 \mathrm{~mol} \%$ of photocatalyst, $20 \mathrm{~mol} \%$ of $\mathrm{Cu}(\mathrm{OAc})_{2}, 1.0$ equiv of DMAP, and $1 \mathrm{~mL}$ of $\mathrm{MeCN} .{ }^{b} \mathrm{NMR}$ yield with 1,3,5-trimethoxybenzene as an internal standard. ${ }^{c}$ Isolated yield.

Received: May 21, 2020

Published: June 25, 2020 
improvements (entry 3). Changing the base additive to quinuclidine or reducing the equivalence of acrylate also led to a reduced yield (entries 4 and 5). On the other hand, the use of a solvent mixture of 1:9 methanol/acetonitrile restored a comparable yield of $80 \%$ for compound 3a (entry 6). In the absence of either photocatalyst or blue light irradiation, only trace amounts of the desired product were detected (entries 7 and 8$)$. Addition of $\mathrm{Cu}(\mathrm{OAc})_{2}$ improved the yield in all cases. A base additive also proved to be crucial to the success of the reaction; without an equivalent of DMAP, no conversion of sulfonyl tetrazole starting material was observed (entry 10).

To further explore the potential of this reaction, a small range of olefinic acceptors (2) as coupling reagents were studied (Figure 1). Benzyl acrylate and tert-butyl acrylate both gave the

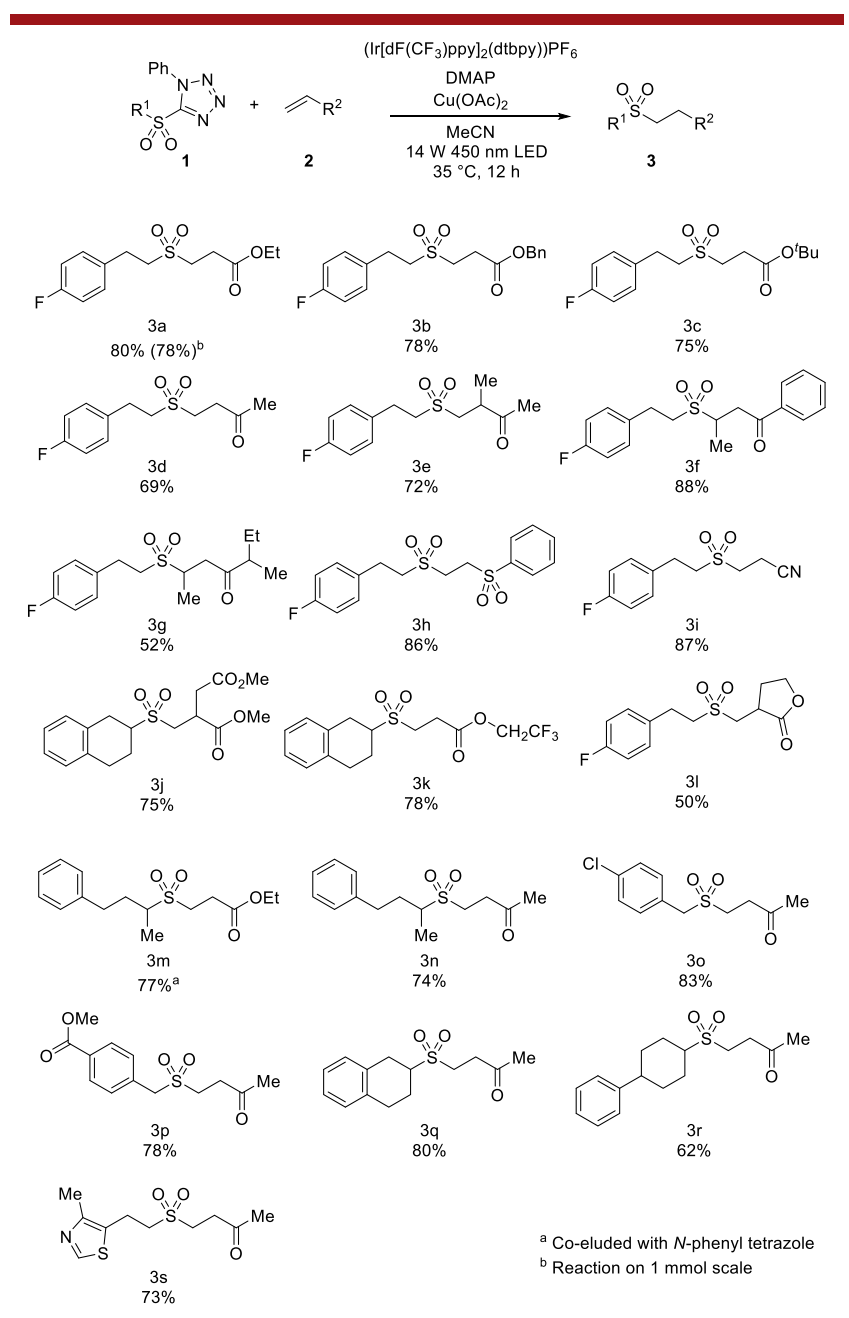

Figure 1. Reaction scope.

corresponding sulfone ( $3 \mathbf{b}$ and $3 \mathbf{c}$ ) in $88 \%$ and $75 \%$ yield, respectively. Vinyl ketone derivatives also delivered satisfying results $(\mathbf{3 d}-\mathbf{g})$, with examples of both terminal (2d and $\mathbf{2 e})$ and internal alkenes ( $2 \mathbf{f}$ and $\mathbf{2 g}$ ). Excellent yields were achieved with vinyl phenyl sulfone as well, with $86 \%$ yield of the bis-sulfone (3h) (as shown by X-ray crystallographic studies, see Supporting Information). Likewise, reaction with acrylonitrile gave the corresponding product (3i) in $87 \%$ yield. Other electron deficient olefins were also able to give the targeted sulfone bearing various functional groups in good yields, such as bis-acetate (3j), trifluoroethyl (3k) and lactone (31). Regarding variations in the sulfone coupling partner, several sulfonyl tetrazoles were prepared and subjected to the optimized conditions. In addition to linear sulfonyl tetrazole 1a, branched tetrazole (1b) was also compatible, giving $77 \%$ of the desired $3 \mathrm{~m}$ and $74 \%$ of $3 \mathrm{n}$. Benzylic sulfones were prepared successfully under the photoredox condition, as demonstrated with examples 3o and 3p. Cyclic branched sulfonyl tetrazoles $\mathbf{1 q}$ and $1 \mathbf{r}$ were also tolerated giving sulfone ( $3 \mathbf{q}$ and $3 \mathbf{r}$ ) smoothly. Finally, heteroaromatic sulfone tetrazole (1s) delivered product 3 s in $73 \%$ yield.

In the absence of copper, the reaction proceeded albeit with a $35 \%$ yield (Table 1 , entry 9 ). On the other hand, it was found essential to have a stoichiometric amount of DMAP present to ensure high yields (Table 1, entry 10). A proposed mechanism to account for these requirements is therefore shown in Figure 2. The Ir(III) catalyst is first excited to a long-lifetime excited

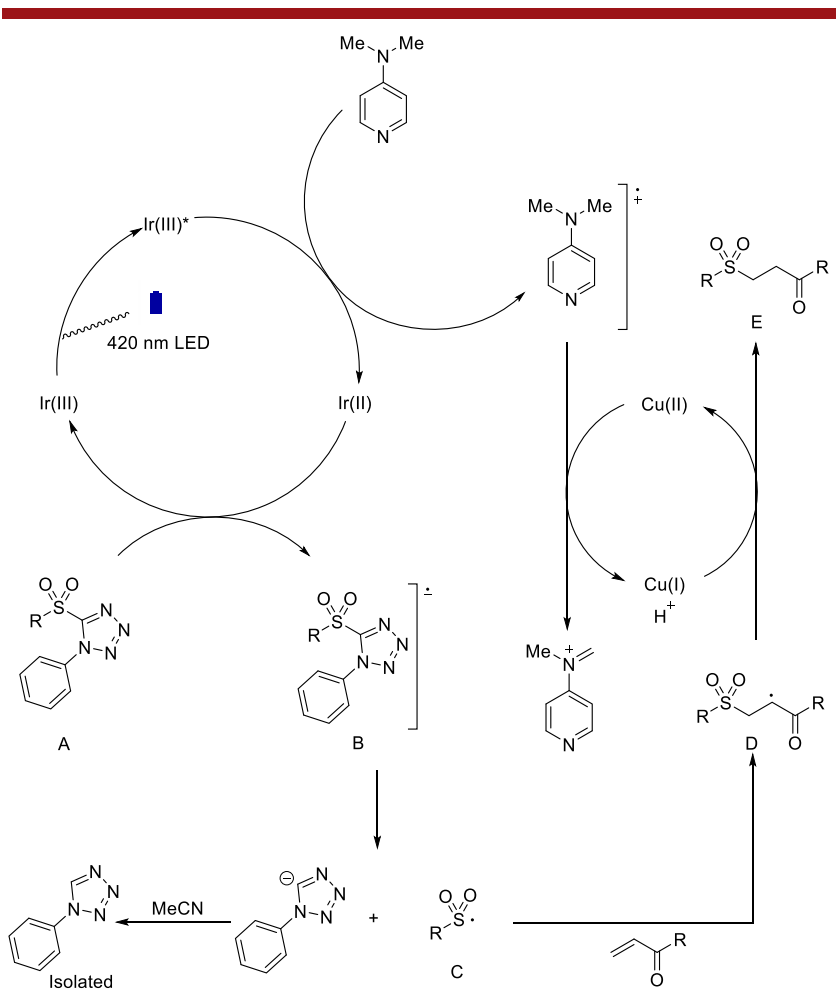

Figure 2. Proposed mechanism.

state $\operatorname{Ir}(\mathrm{III}) *$ when exposed to blue light. ${ }^{13}$ Following this, DMAP undergoes a single electron oxidation process, resulting in a DMAP radical cation and the reduced $\operatorname{Ir}(\mathrm{II})$ species $\left(E_{1 / 2}\right.$ Ox $=1.21 \mathrm{~V}$ vs SCE) ${ }^{14}$ The radical cation could then be quenched by a $\mathrm{Cu}$ (II) species to an iminium cation, which we believe was lost due to workup by absorption on silica gel chromatography. ${ }^{15}$ In the reduction step, sulfone tetrazole $\mathbf{A}\left(E_{1 / 2}{ }^{\text {Red }}=\right.$ $-1.31 \mathrm{~V}$ vs SCE, see Supporting Information) is reduced by $\operatorname{Ir}(\mathrm{II})$ complex $\left(E_{1 / 2}{ }^{\text {Red }}=-1.37 \mathrm{~V}\right.$ vs SCE$)$ via single electron reduction giving a radical anion $\mathbf{B}$, where the $\mathrm{C}-\mathrm{S}$ bond breaks to provide the sulfone radical $\mathrm{C}$ and $\mathrm{N}$-phenyl tetrazole anion. A similar photoinduced bond-breaking process was noted by both the Baran group ${ }^{11}$ and Kamijo et al. respectively. ${ }^{16}$ The $N$ phenyl tetrazole anion is neutralized and the sulfone radical is trapped by olefinic acceptors to form radical $D$, which is quenched by the previously generated $\mathrm{Cu}(\mathrm{I})$ complex to give the corresponding anion and neutralized to the final product sulfone $\mathbf{E}$. In many cases, the copper catalyst at the end of the reaction was deposited as a $\mathrm{Cu}(0)$ mirror on the surface of the 
flask, thus suggesting it plays a role as an oxidant in these reactions. The possibility also could not be ruled out that the copper species, namely $\mathrm{Cu}(\mathrm{II})$ and $\mathrm{Cu}(\mathrm{I})$ complexes, ${ }^{17}$ were involved in the initial oxidation process of DMAP. ${ }^{18}$

In summary, we have demonstrated that sulfone-substituted $\mathrm{N}$-phenyltetrazoles undergo efficient photoredox coupling with acrylates and related electron-withdrawing olefinic acceptors to give coupled products retaining the sulfone moiety, suggesting the intermediacy of sulfonyl radicals. Reaction yields are generally very good and occur with high substrate tolerance of functionality. The products of these reactions could find useful applications in medicinal chemistry programs ${ }^{19}$ or as precursors in further Ramberg-Bäcklund reactions, for example.

\section{ASSOCIATED CONTENT}

\section{SI Supporting Information}

The Supporting Information is available free of charge at https://pubs.acs.org/doi/10.1021/acs.orglett.0c01730.

Details of experimental procedure, compound characterization data, and NMR spectra (PDF)

\section{Accession Codes}

CCDC 1998286 contains the supplementary crystallographic data for this paper. These data can be obtained free of charge via www.ccdc.cam.ac.uk/data_request/cif, or by emailing data request@ccdc.cam.ac.uk, or by contacting The Cambridge Crystallographic Data Centre, 12 Union Road, Cambridge CB2 1EZ, UK; fax: +44 1223 336033. Additional data related to this publication is available at the University of Cambridge Institutional Data Repository 10.17863/CAM.51612.

\section{AUTHOR INFORMATION}

\section{Corresponding Author}

Steven V. Ley - Department of Chemistry, University of

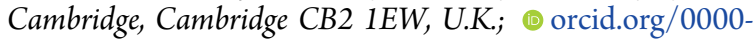
0002-7816-0042; Email: svl1000@cam.ac.uk

\section{Authors}

Yiding Chen - Department of Chemistry, University of Cambridge, Cambridge CB2 1EW, U.K.; orcid.org/00000001-6932-9005

Nicole McNamara - Faculty of Pharmacy and Pharmaceutical Sciences, Monash Institute of Pharmaceutical Sciences, Monash University (Parkville Campus), Parkville, VIC 3052, Australia

Oliver May - Department of Chemistry, University of Cambridge, Cambridge CB2 1EW, U.K.

Thanigaimalai Pillaiyar - Department of Chemistry, University of Cambridge, Cambridge CB2 1EW, U.K.; Pharmaceutical Institute, Department of Pharmaceutical and Medicinal Chemistry, University of Bonn, 53121 Bonn, Germany; (1) orcid.org/0000-0001-5575-8896

David C. Blakemore - Medicine Design, Pfizer, Inc., Groton, Connecticut 06340, United States

Complete contact information is available at: https://pubs.acs.org/10.1021/acs.orglett.0c01730

\section{Notes}

The authors declare the following competing financial interest(s): D.C.B. is an employee and stockholder of Pfizer Inc.

\section{ACKNOWLEDGMENTS}

We thank Dr. Andrew Bond of University of Cambridge for conducting X-ray crystallography experiments and Dr. Steve Brenek of the Pfizer Groton Process Safety Group for supplying DSC data commentary. Y.C. thanks Pfizer for funding the postdoctoral fellowship. T.P. thanks COST Action CA18133 ERNEST for funding short term scientific mission (STSM). We thank the American Chemical Society through the Arthur C. Cope Fund (award to S.V.L.). The authors also gratefully acknowledge financial support from H2020-FETOPEN-20162017 programe of European commission (S.V.L; Grant Agreement No. 737266-ONE FLOW).

\section{REFERENCES}

(1) Milligan, J. A.; Phelan, J. P.; Badir, S. O.; Molander, G. A. Angew. Chem., Int. Ed. 2019, 58, 6152-6163.

(2) Zhu, J.; Yang, W.-C.; Wang, X.-d.; Wu, L. Adv. Synth. Catal. 2018, 360, 386-400.

(3) Scott, K. A.; Njardarson, J. T. Topics Curr. Chem. 2018, 376, 5.

(4) Chaudhary, R.; Natarajan, P. ChemistrySelect 2017, 2, 6458-6479.

(5) Bagal, D. B.; Kachkovskyi, G.; Knorn, M.; Rawner, T.; Bhanage, B. M.; Reiser, O. Angew. Chem., Int. Ed. 2015, 54, 6999-7002.

(6) (a) Liu, N.-W.; Hofman, K.; Herbert, A.; Manolikakes, G. Org. Lett. 2018, 20, 760-763. (b) Wang, J.-J.; Yu, W. Org. Lett. 2019, 21, 9236-9240.

(7) Yang, D.; Huang, B.; Wei, W.; Li, J.; Lin, G.; Liu, Y.; Ding, J.; Sun, P.; Wang, H. Green Chem. 2016, 18, 5630-5634.

(8) (a) Hell, S. M.; Meyer, C. F.; Laudadio, G.; Misale, A.; Willis, M. C.; Noël, T.; Trabanco, A. A.; Gouverneur, V. J. Am. Chem. Soc. 2020, 142, 720-725. (b) Hell, S. M.; Meyer, C. F.; Misale, A.; Sap, J. B. I.; Christensen, K. E.; Willis, M. C.; Trabanco, A. A.; Gouverneur, V. Angew. Chem., Int. Ed. 2020, DOI: 10.1002/anie.202004070.

(9) Wei, J.; Bao, K.; Qi, C.; Liu, Y.; Ni, C.; Sheng, R.; Hu, J. Adv. Synth. Catal. 2019, 361, 5528-5533.

(10) (a) Aïssa, C. Eur. J. Org. Chem. 2009, 2009, 1831-1844. (b) Plesniak, K.; Zarecki, A.; Wicha, J. In Sulfur-Mediated Rearrangements II; Schaumann, E., Ed.; Springer: Berlin, Heidelberg, 2007; pp $163-250$.

(11) Merchant, R. R.; Edwards, J. T.; Qin, T.; Kruszyk, M. M.; Bi, C.; Che, G.; Bao, D.-H.; Qiao, W.; Sun, L.; Collins, M. R.; Fadeyi, O. O.; Gallego, G. M.; Mousseau, J. J.; Nuhant, P.; Baran, P. S. Science 2018, $360,75-80$.

(12) It was worth noting that some sulfone tatrazoles, especially with small molecular weight, have exotherm under elevated temperature $\left(>150{ }^{\circ} \mathrm{C}\right)$ in their neat form. Hence, extra caution should be taken while handling these materials.

(13) Lowry, M. S.; Goldsmith, J. I.; Slinker, J. D.; Rohl, R.; Pascal, R. A.; Malliaras, G. G.; Bernhard, S. Chem. Mater. 2005, 17, 5712-5719.

(14) Skubi, K. L.; Blum, T. R.; Yoon, T. P. Chem. Rev. 2016, 116, 10035-10074.

(15) (a) Rueping, M.; Koenigs, R. M.; Poscharny, K.; Fabry, D. C.; Leonori, D.; Vila, C. Chem. - Eur. J. 2012, 18, 5170-5174. (b) Freeman, D. B.; Furst, L.; Condie, A. G.; Stephenson, C. R. J. Org. Lett. 2012, 14, 94-97.

(16) Kamijo, S.; Kamijo, K.; Murafuji, T. Synthesis 2019, 51, 38593864.

(17) Kratochvil, B.; Zatko, D. A.; Markuszewski, R. Anal. Chem. 1966 $38,770-772$.

(18) Lima, F.; Sharma, U. K.; Grunenberg, L.; Saha, D.; Johannsen, S.; Sedelmeier, J.; Van der Eycken, E. V.; Ley, S. V. Angew. Chem., Int. Ed. 2017, 56, 15136-15140.

(19) Trost, B. M.; Kalnmals, C. A. Chem. - Eur. J. 2019, 25, 1119311213.

\section{NOTE ADDED AFTER ASAP PUBLICATION}

Figure 1 and the Supporting Information file were replaced on July 15, 2020. 